failed to respond to oxacillin but recovered when gentamicin was given in addition, a pointer to the type of treatment that may be necessary in such cases.) Sabath and his colleagues ${ }^{2}$ determined the concentrations of nafcillin respectively inhibiting and killing their strains after both 24 and 48 hours. The difference in these concentrations at 24 hours varied from 128- to 2000-fold; at 48 hours it fell to nil or only 4-fold for three strains, but remained high or relatively high for the remainder.

These results imply that some tolerant strains are simply killed more slowly and some not at all. Mayhall et $a l^{3}$ found more encouraging results from 48-hour tests of bactericidal action, using a rather different method. Sabath et $a l^{2}$ also found tolerance to cephalothin and vancomycin but not to gentamicin, cycloserine, or rifampicin and confirmed reduced autolysin production, as reported by Best et al. ${ }^{1}$ Nor is tolerance a rarity: it was found in $28\left(44^{\circ}{ }_{0}\right)$ of 63 strains from blood cultures in the University of Minnesota hospitals in the past year, while Mayhall et $a l^{3}$ working in St Louis, Missouri, found 33 out of 60 strains to be tolerant. Perhaps as many as one-half of all serious staphylococcal infections may now respond poorly to any penicillin, so that a test of bactericidal action should be included in routine methods. Possibly tests of combined bactericidal action with aminoglycosides may be required in future to direct effective treatment; simplified methods for performing such tests would be welcome.

Another hitherto unrecognised obstacle to effective penicillin treatment is reported for the first time in two papers at pp 991 and 998. This is the presence in an inflammatory exudate of something which destroys penicillin. It has long been known that penicillinase may be formed by some resistant organism present in addition to the sensitive pathogen against which treatment is directed; much has been written, for example, on the possible effect of penicillinase-forming staphylococci in the throat on the action of penicillin in the prophylaxis of rheumatic fever. In treating acute streptococcal throat infections when resistant staphylococci are present oxacillin (resistant to penicillinase) succeeds when penicillin has failed. "Another example is the "indirect pathogenicity," as it was called by Maddocks and May, of penicillinase-forming enterobacteria in the bronchial secretion, shown in six patients to inactivate ampicillin given for $H$ influenzae infection. What has never been reported before is the presence in an inflammatory exudate of an enzyme destroying penicillin derived not from bacteria but apparently from host cells. Proof of such an origin seems clear in the patient described by Barnes and Waterworth on $\mathrm{p} 991$. Their patient had an empyema due to a fully penicillin-sensitive group B Streptococcus pyogenes. Large doses of penicillin both intramuscularly and injected into the empyema cavity itself were without effect, and not only did the pus contain little or no penicillin despite this vigorous treatment, but it inactivated penicillin added to it in vitro. It also inactivated, though at varying rates, four other penicillins and seven cephalosporins but had no effect on various unrelated antibiotics, to one of which the patient later responded promptly. Several reasons are given for concluding that the inactivating agent was an enzyme, and fractionation of the pus indicated its derivation from the cells.

This cannot be dismissed as a wholly exceptional occurrence, since by coincidence de Louvois and Hurley ( $p$ 998) had been engaged independently on a study of methods for the assay of antibiotics in pus, and among 22 specimens found four which inactivated penicillin, ampicillin, and cephaloridine in the same way. Their similar studies of the nature and origin of the inactivating agent point to the same conclusions. Here, then, is another possible reason for penicillin treatment failure which is well worth bearing in mind and certainly merits further investigation to determine what this enzyme may be and what determines its formation.

${ }^{1}$ Best, G K, Best, N H, and Koval, A V, Antimicrobial Agents and Chemotherapy, 1974, 6, 825.

Sabath, L D, et al, Lancet, 1977, 1, 443.

${ }^{3}$ Mayhall, C G, Medoff, G, and Marr, J J, Antimicrobial Agents and Chemotherapy, 1976, 10, 707.

${ }^{4}$ Simon, H J, and Sakai, W, Pediatrics, 1963, 31, 463.

5 Maddocks, J L, and May, J R, Lancet, 1969, 1, 793.

\section{Postinfarction ventricular aneurysms}

Discussion of ventricular aneurysms is complicated by the lack of any uniform definition. At operation or necropsy an aneurysm is recognised as an area of thin, noncontractile fibrous tissue, well demarcated from the surrounding myocardium. A further distinguishing feature of an aneurysm should be that it bulges outwards from the ventricular wall; if it does not it is simply a fibrous scar. ${ }^{1}$ In postmortem series aneurysms have been recorded in $3.5 \%$ to $20 \%$ of previously infarcted hearts, ${ }^{2}$ but there may be more which have a good prognosis.

Aneurysms are nearly always associated with total occlusion of the coronary artery, usually the left anterior descending, supplying the affected myocardium. There are no pathognomonic clinical features. The most common symptoms are dyspnoea due to left ventricular failure and angina. Recurrent arrhythmias and systemic emboli are also more frequent than in patients with uncomplicated infarction. On examination there may be a high, sustained ventricular impulse with added third or fourth heart sounds. A persistent rise in the ST segment on the electrocardiogram is suggestive but not conclusive evidence of an aneurysm. The chest $x$-ray film shows simple enlargement of the heart more often than the characteristic bulge on the lateral border of the heart shadow. Calcification may occasionally be seen in the aneurysm wall.

When the clinical history and the ECG suggest the possibility of aneurysm the essential investigation is left ventricular angiography with coronary arteriography. The aneurysm will appear as a segment of wall which does not move (akinetic) or appears to bulge out, paradoxically, in systole (dyskinetic). Unfortunately diffusely scarred and ischaemic areas of wall may also appear dyskinetic, and surgical resection may not be warranted in these cases. Surgery is indicated if there is persistent failure, angina, uncontrollable ventricular arrhythmias, or systemic emboli (anticoagulants are of no proved value in this last group of patients). In assessing a patient for surgery it is important to establish how well the remaining myocardium is contracting and whether the pattern of coronary disease is amenable to bypass grafting.

Donaldson $e t a l^{4}$ recently reported the results of surgery in 32 patients. In 25 dyspnoea was the main symptom, in six it was angina, and one had recurrent supraventricular tachycardia. Nineteen patients had persistent ST elevation and 28 had enlargement of the heart on the chest radiograph. The left ventricular angiogram showed dyskinesia in 30 of the 32 cases, and 30 patients had severe disease of one coronary 
vessel or more. At operation all patients had the aneurysm resected, and 18 had saphenous vein bypass grafts or other additional procedures. There were two surgical deaths and one death at six months, all in patients with severe disease. Of the 29 survivors after six to 56 months' follow-up, 18 were leading a normal life on no drugs, five were asymptomatic on treatment, and three had dyspnoea. Two patients had persistant angina; in one of these the graft had blocked.

These results show how good the symptomatic benefit may be in selected patients-though many of the patients had additional procedures. Ultimate prognosis may also be affected: in a large series from Cleveland ${ }^{5}$ the four-year survival in patients treated by operation was $76 \%$ compared with a five-year survival of less than $20 \%$ in those who were treated medically. Death was usually due to reinfarction or intractable failure, and hardly ever to rupture of the aneurysm. Clearly the two groups were not comparable, since the surgical patients were highly selected.

When a patient develops persistent failure or angina, or both, after an infarct (especially if it is his first) some mechanical complication should be suspected. If ventricular septal defect and mitral regurgitation have been excluded, then an aneurysm should be sought. In well-chosen cases surgical treatment provides good symptomatic relief and may well prolong life.

${ }^{1}$ Edwards, T E, An Atlas of Acquired Diseases of the Heart and Great Vessels, vol 2, p 615. Philadelphia, W B Saunders, 1961.

2 Dubnow, M H, Burchell, H B, and Titus, J L, American Heart fournal, $1965,70,753$.

3 Schlichter, J, Hellerstein, H K, and Katz, L N, Medicine, 1954, 33, 43.

4 Donaldson, R M, et al, British Heart fournal, 1976, 38, 1223.

${ }^{5}$ Loop, F D, et al, Annals of Surgery, 1973, 178, 399.

\section{Topical corticosteroids and relapses of psoriasis}

Many patients have psoriasis so mild that they hardly need to seek medical advice; in others it may ruin and even threaten their lives. Sometimes the disease runs an unfluctuating course over many years; more usually there are definite fluctuations from time to time, often for no apparent reason and unrelated to factors such as infections, trauma, season, or emotions. We know little about the long-term history, with or without treatment, in the mass of psoriatics, partly because most series (with a few exceptions ${ }^{1}$ ) are based on hospital statistics. The duration and completeness of remissions induced by treatment are surely just as important as how quickly they are achieved.

For the relatively few really bad cases of psoriasis unresponsive to conventional inpatient treatment potent drugs such as methotrexate, hydroxyurea, and other antimitotic agents are both justified and effective. ${ }^{2}{ }^{3}$ There is virtually no place for systemic corticosteroids. The recently introduced PUVA therapy ${ }^{4}$ long-wave ultraviolet light-is gaining popularity, but until the long-term side effects have been assessed it should be reserved for the very severe cases, and in any case such treatment is not generally available in Britain.

Patients with ordinary psoriasis are usually treated with topical applications of tar, dithranol, or corticosteroids, perhaps combined with ultraviolet light, either as outpatients or more effectively as inpatients. ${ }^{2} 6$ Healing rates with different regimens have been compared often and in detail, and it seems that topical steroids act more quickly, are cleaner and therefore more popular, but often fail to achieve complete clearance. They tend to fail in the more difficult patients with widespread or inflamed psoriasis. Persistence with steroid treatment in such cases has almost certainly been responsible for the very definite increase in the past 20 years of the more unpleasant types such as pustular psoriasis. ${ }^{7}$ The relapse rate after various topical treatments is far less well known. Over ten years ago patients given fluocinolone under occlusion were found to relapse more quickly than those given conventional tar treatment, ${ }^{8}$ so it seemed reasonable next to try a combination of tar or dithranol with a topical steroid, which might allow the best of both worlds. A recent retrospective comparison $^{9}$ of psoriatics treated as inpatients with topical betamethasone valerate under polyethylene occlusion combined with dithranol and another group treated with dithranol alone showed that the rate of clearance in the two groups was comparable, the group given dithranol alone taking marginally longer. Nevertheless, the relapse rate was strikingly different: the mean relapse time was five weeks in those given steroids plus dithranol and 28 weeks in those given dithranol alone. Such studies are hard to organise, but further long-term trials, preferably prospective, are needed.

Should these findings be extrapolated to other topical steroids or to steroids without polyethylene occlusion ? Weak nonfluorinated steroids give disappointing results in psoriasis. Very strong steroids such as clobetasol propionate achieve immediate clearance of psoriasis even without polyethylene, but it might be thought that they would have even greater potential for damage to the skin, to the pituitary-adrenal axis, and to the long-term natural history of the disease. Nevertheless, in a small series of patients Marriott and Munro ${ }^{10}$ found that the relapse rate after clobetasol was slower than after dithranol.

For the moment the conclusion must be that the use of topical steroids is no panacea or even the best long-term management for most patients with psoriasis. They are useful in localised psoriasis, but not indefinitely, and steroids must not be used in increasing quantities in the face of clinical deterioration. In long-term treatment the dose of strong steroids should be kept below $25 \mathrm{~g}$ weekly and the clinician should watch for early signs of skin atrophy. In more extensive cases of psoriasis the temptation to use topical steroids for long periods must be resisted. Some of these patients may improve a lot when given a strong steroid for up to six weeks, and then do not necessarily relapse when the steroid is stopped (which should be done gradually, replacing with a weaker steroid or with the same one diluted). If they do relapse an alternative treatment must be sought. Topical use of dithranol or tar is still worth while, and both become much more powerful remedies when used in inpatients. There is still truth in the dictum that the more powerful drugs should not be used until conventional inpatient treatment has been proved wanting.

${ }^{1}$ Lomholt, G, Psoriasis. Copenhagen, Gad, 1963.

${ }^{2}$ Dahl, M G C, British Medical fournal, 1971, 3, 234.

${ }^{3}$ Farber, E M, Pearlman, D, and Abel, E A, Archives of Dermatology, 1976, $112,1679$.

4 Wolf, K, et al, Archives of Dermatology, 1976, 112, 943.

${ }^{5}$ British Medical fournal, 1975, 1, 474.

6 Baker, H, Dermatologica, Basel, 1975, 150, 136.

${ }^{7}$ Baker, H, British fournal of Dermatology, 1976, 94, suppl 12, 83.

${ }^{8}$ Knudsen, E A, Acta Dermatovenereologica, 1965, 45, 50.

${ }^{9}$ Seville, R H, British fournal of Dermatology, 1976, 95, 643.

${ }_{10}$ Marriott, P J, and Munro, D D, British fournal of Dermatology, 1976, 94, suppl 12, 101 